
\title{
25 Research Square \\ Tai Chi versus High-intensity Interval Training on Inhibitory Control in Individuals with Substance Use Disorder
}

\section{Xiaowu Pang}

Shanghai University of Sport

Dong Zhu ( $\nabla$ zhudong@sus.edu.cn )

Shanghai University of Sport

\section{Suyong Yang}

Shanghai University of Sport

\section{Xiao Ke}

Shanghai Drug Administration

\section{Tianyuan Wang}

Shanghai University of Sport

\section{Jiabin Wang}

Shanghai University of Sport

\section{Wolfgang I. Schöllhorn}

Johannes Gutenberg University of Mainz

Yanqiang Yin

Zhejiang Sci-Tech University

\section{Research Article}

Keywords: Tai chi, High-intensity interval training, Acute exercise, Substance use disorder, Cognitive rehabilitation, Methamphetamine

Posted Date: March 9th, 2021

DOl: https://doi.org/10.21203/rs.3.rs-296322/v1

License: (c) (i) This work is licensed under a Creative Commons Attribution 4.0 International License. Read Full License 


\section{Abstract \\ Background}

Cognitive function of individuals with substance use disorder (SUD) is defective, and higher cognitive inhibitory control ability is considered to be related to lower drug craving. As a conjunction treatment for individuals with SUD, exercise has received supports and attention in many countries recently. Exercise has been reported to be beneficial for cognitive rehabilitation. However, different exercises have different effects on cognitive function. The purpose of this study is to investigate the effects of a single session of Tai chi (TC) exercise and high-intensity interval training (HIIT) on inhibitory control in individuals with SUD.

\section{Methods}

A total 47 individuals with methamphetamine dependence were recruited from a compulsory drug rehabilitation center; participation in this study was voluntary. The participants were randomly assigned to the TC group or the HIIT group, and computer-based Go/No-go and Stroop tasks were used to assess inhibitory control in an indoor setting prior to and following exercise. Independent sample ttest was applied for baseline comparison of continuous variables, while repeated-measures analysis of variance was applied to test differences in the effect of each intervention before and after a single session of exercise.

\section{Results}

In Go/No-go test, the reaction time of the TC and HIIT groups in the post-test was shorter than that at the baseline, and the response accuracy of the post-test were higher than that of the baseline. In the Stroop task, the reaction time of two groups in the post-test was shorter than that at the baseline; while, greater improvement in response accuracy was observed in HIIT group in the post-test than that of the baseline.

\section{Conclusion}

Both TC and HIIT can promote inhibitory control in individuals with SUD. Compared with the TC group, the HIIT group showed greater improvements in response accuracy. These findings demonstrate the potential of TC and HIIT in improving cognition in SUD.

\section{Trial registration}

: ChiCTR1900022158 Chinese Clinical Trial Registry: Registered 27th March, 2019. 


\section{Introduction}

As a global public health problem [1], substance use disorder (SUD) has elicited increasing concern. Complex reasons may explain why individuals with SUD are difficult to cure. Numerous studies have demonstrated that SUD or drug addiction is a chronic and relapsing brain disease in which addicted patients continually exhibit drug-seeking behavior despite its adverse consequences for their psychology and physiology [2-4]. Neuropsychological studies have verified that the cognitive function of individuals exposed to certain substances (e.g., drugs, alcohol, cigarettes) for extended periods of time show significant defects that can be associated with abnormal brain function and metabolism caused by substance addiction $[5,6]$. The cognitive impairment of drug addicts is mainly manifested by their higher level of impulsive behavior and decision-making compared with the normal population [7, 8]. Compared with assessments obtained after recent methamphetamine (Meth) use, the impulsivity self-reported by Meth-dependent subjects was significantly higher following approximately 1 week of abstinence from drugs [7]. Elevated impulsivity and impaired decision-making cognition have been reported in heavy drug users; both conditions are strongly state-dependent in a SUD population and may be suitable for monitoring treatment success $[9,10]$. A number of studies have confirmed the presence of learning and memory disorders in drug addicts, and these disorders have been proposed to increase the vulnerability of the latter to drug addiction $[11,12]$. That is to say, learning and memory defects render individuals likely to become addicted to drugs and increase the difficulty of abstinence. Therefore, improving the cognitive function of drug addicts can be assumed to play a vital role in drug rehabilitation.

Physical exercise has been strongly recommended as a rehabilitation program for SUD $[6,13]$ and has been proven to be a positively effective means of drug rehabilitation [14]. Numerous studies have assessed the effects of short exercise sessions and long-term exercise protocols as an adjunct therapy in SUD. The majority of these studies focus on physical and mental health, craving, duration of abstinence, and withdrawal symptoms [15-20]. The impacts of exercise on cognitive psychology and brain neuroscience have received growing interest in efforts to explore the mechanism of drug addiction. Studies have shown that exercise can improve age-related declines in cerebral blood flow, which is associated with improved cognitive function [21]. The beneficial effects of long-term exercise on cognition has been demonstrated [14, 22], and even a single session of aerobic exercise (i.e., acute exercise) has been consistently shown to exert a positive influence on cognitive function [23]. Acute exercise effectively improves inhibition control in individuals with SUD [24]. However, despite the numerous positive effects of exercise on the human body, systematic research on the application of this therapy to individuals with SUD remains at an early stage. Studies in this area are assigned the strictest criteria of scientific rigor, and the related mechanisms and modalities have yet to be thoroughly investigated [25]. Most available studies solely explore the effectiveness of aerobic or moderate-intensity exercise on the physical attributes, mental recovery, and brain function of patients with SUD. While previous studies have shown benefits in motoric and cognitive domains, how these effects are functionally related remains unclear. The effectiveness of different doses of specific exercise parameters (e.g., intensity) must also be investigated [20], as this topic seldom discussed in research on exercise rehabilitation for drug addicts [26]. Some studies have explored the effects of moderate- and high- 
intensity exercise, as well as aerobic and anaerobic forms of exercise, on drug rehabilitation [25, 27]. However, the single-feature (e.g., moderate intensity) exercise protocols used therein [26] do not allow precise exercise prescriptions to be developed for individuals with SUD.

Tai chi (TC), a traditional Chinese sport, has been increasingly applied to the field of drug rehabilitation. Previous studies reported that TC can be beneficial to the physical and mental health of individuals with SUD $[17,18]$, but how TC affects the cognition of SUD is not completely understood. A large number of studies have proven that TC can improve cognition in the elderly and cognitive-impairment groups [28]. The cognitive impairment of drug addicts has been confirmed [5], and the results provide indications for the improvement of cognitive function, especially improvement of inhibition control, are related to drug abstinence $[11,12]$. A greater understanding of the cognitive impact of TC on patients with SUD is warranted.

Depending on its style, TC is described as a form of physical exercise with low to moderate intensity [18, $29,30]$. In general, a TC style with moderate intensity is believed to assist detoxification, relieve withdrawal syndrome, and inhibit relapse impulses and behaviors; it is also considered relatively safe [31-33]. Moderate-intensity continuous training (MICT) is widely used in drug rehabilitation programs [22]. The effects of high-intensity interval training (HIIT) on drug addicts have received great attention [27] as HIIT is considered a time-saving, highly efficient, and economical form of exercise [34]. HIIT is derived from the Tabata protocol [35] and characterized by a short series of high-intensity repetitions interspersed with low-intensity recovery phases. However, increased exercise intensity is widely believed to result in greater risk of adverse events, such as muscle injury and systemic inflammation [36-38]. The application of high-intensity exercise to drug dependents is considered a critical endeavor because patients with SUD are not normally in excellent physical condition, $[17,18]$ and participation in high-intensity sport features an inherent risk of sports-related injuries [39]. Nevertheless, several long- and short-term studies argue that HIIT has the same value as, if not is better than, MICT in terms of improving body composition, cardiovascular function, metabolic health tolerance, safety, and exercise enjoyment and adherence [4045]. More importantly, some authors have emphasized that HIIT is beneficial in reducing cardiovascular risk and indirectly improving mortality risk $[27,46]$. Unlike TC or other moderate exercise, HIIT may require more exercise monitoring and specific patterns to reduce potential risks because high-intensity exercise may be challenging for the SUD population. Different exercise intensities or forms could incite different expectations of cognitive benefits. Sport-physiological research supports the premise that higher-intensity exercise treatments lead to greater cognitive benefits compared with moderate-intensity exercise [47]. HIIT has been reported to have a positive effect on improving cognition in the elderly and cognitiveimpairment groups [48-50]. Currently, the evidence in favor of high-intensity training for SUD is weak. Few studies have discussed the cognitive rehabilitation of patients with SUD through high-intensity training $[26,27,51]$. Consequently, the impact of HIIT on the cognition of patients with SUD is unclear and requires further research.

Whether different forms or intensities of exercise exert different effects on substance use-related outcomes must be determined. The theoretical basis of TC and HIIT beside the cognitive treatment of 
SUD has not been fully confirmed in the scientific literature. Given the benefits of TC and HIIT in physical and cognitive rehabilitation in different groups, this study seeks to explore which form of exercise is more suitable for the cognitive rehabilitation of SUD. The purpose of this study is to compare the effects of TC and HIIT on the recovery of cognitive inhibition control in patients with SUD by using specific cognitive tests that are typically associated with executive functions.

\section{Materials And Methods}

\subsection{Design}

This single (assessor)-blind, two-group randomized controlled trial was conducted to compare the acute effects of TC and HIIT practice. The study was carried out from November 2019 to December 2019 and included a single session of TC or HIIT intervention. The primary outcome was measured at the baseline and after each session. All exercise interventions and tests were conducted in a laboratory environment. Participants were recruited from a 1-year follow-up trial including individuals participating in either highintensity exercise (i.e., running, resistance training, rope skipping) or TC [18] for at least 6 months in a Shanghai compulsory rehabilitation center (SCRC). The frequency of exercise was 3 times a week, and each session was conducted for 1 hour.

The study protocol was approved by the Ethical Committees of the Shanghai University of Sport and the Shanghai Narcotics Control Commission.

All subjects signed written informed consent forms prior to participating in this study.

\subsection{Participants}

The participants were male Meth-dependent individuals. At the time of recruitment, a total of 120 individuals with SUD were receiving treatment in an SCRC; of these, 47 eligible Meth-dependent individuals voluntarily participated in this study. The inclusion criteria were: 1) age 18-40 years, 2) met the diagnosis of Meth dependence according to Diagnostic and Statistical Manual of Mental Disorders criteria (DSM-IV), 3) treatment duration in the SCRC exceeding 1 year; 4) no serious medical or mental illness, and 5) completed primary school or a higher level of education. The exclusion criteria were: 1) currently diagnosed with a disease of the cardiovascular system, respiratory system, or nervous system; 2) anti-social personality disorder or borderline personality disorder; and 3) unwilling to accept the assigned intervention conditions. All participants completed a demographic questionnaire and physical fitness test. Table 1 presents the participant demographics.

\subsection{Cognitive tasks}

Go/No-go task. A modified Go/No-go task in which two different stimuli (inverted triangle-No-go stimulus or positive triangle-Go stimulus; side length, $7 \mathrm{~cm}$ ) were focally presented on a computer screen with a gray background (brightness, $60 \mathrm{CD} / \mathrm{m}^{2}$ ) was employed. The stimulus sequence consisted of 200 stimuli and showed inverted or positive triangles with equal probability. One stimulus appeared randomly in each trial, and the number of consecutive occurrences of each same stimulus was less than four times. The 
stimuli were presented for 100 ms with a variable inter-stimulus interval (1000-2000 ms; average, 1500 $\mathrm{ms}$ ) to eliminate the expectation effect of the subjects. The subjects were asked to use the thumb button as soon as possible when presenting the positive triangle (Go stimulus) but not when presenting an inverted triangle (no go stimulus).

Stroop task. A modified computerized Stroop test [52] was used in this study. The stimuli were four

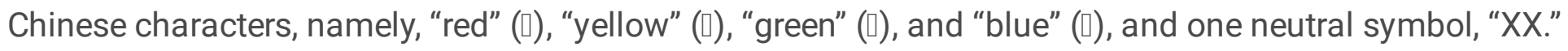
Two conditions, namely, congruent and incongruent, were established. In the congruent condition, the font color was identical to the character meaning or symbol; in the incongruent condition, the font color was different from the character meaning or symbol. The subjects were asked to respond to the color of the characters or symbols and ignore the meaning of the words.

Participants provided their responses by response key mapping (i.e., red, A key; yellow, S key; green, K key; blue, L key).

In the experiment, each participant was allows 240 trials. Four color words and four colored symbols were presented 30 times in random order. The subjects were required to distinguish the color of the stimulus and make the corresponding key reaction on the premise of ensuring the correct response. The stimulating words were displayed on a computer screen for $1000 \mathrm{~ms}$ and disappeared when the response key was pressed. The next stimulus was displayed after $500 \mathrm{~ms}$.

Participants did not speak during the Go/No-go and Stroop tasks to avoid anthropic factors resulting from speaking.

Reaction time (RT) and response accuracy were recorded for all tasks. Cognitive tasks were programmed by E-prime 3.0 (Psychology Software Tools, Inc, Sharpsburg, PA, USA).

Recent studies have suggested that the Go/No-go and Stroop tasks could be used to evaluate inhibitory control $[8,52,53]$. The Go/No-go task focuses on measuring motor response inhibition, while the Stroop task measures a more cognitive form of inhibition called interference inhibition [54]. Most of the available studies employed RT and response accuracy as the primary measures of interest [8,55-57]. Taking the Go/No-go task test as an example, subjects must respond to "Go" stimuli quickly while suppressing responses to "No-go" stimuli accurately; this task requires inhibitory control to overcome the trend of automatic response. In this paradigm, RT refers to the average RT of all correct stimuli, and response accuracy refers to the number of stimuli to which the correct response was given relative to the total number of stimuli. A shorter RT or higher response accuracy or both indicates stronger inhibitory control [8].

\subsection{Exercise intervention}

The participants were randomly assigned by a computer to the TC $(n=24)$ or the HIIT group $(n=23)$ and participated in a single session of exercise. The participants were equipped with a heart rate (HR) monitor (Polar TeamPro, Finland) and then engaged in a $30 \mathrm{~min}$ session of TC or HIIT. The TC protocol consisted of a 5 min warm-up, 20 min of modified TC [18] at 55-75\% maximum heart rate $\left(\mathrm{HR}_{\max }\right)$, and a 5 min 
cool-down. The HIIT protocol consisted of a 5 min warm-up, 20 min of interval exercise, and a 5 min cooldown. The participants performed warm-up and cool-down at a self-determined speed on a treadmill ( $\mathrm{SH}$ 5921 , Shangqiu, China) and completed five cycles of 2 min of running at $85-95 \% \mathrm{HR}_{\max }$ (estimated as 220 - age) separated by 2 min of self-paced walking. Participants in the HIIT group were allowed to ask the experimenter to change the running speed if they felt it necessary. Ten HR indicators were recorded every 2 min during the 20 min exercise phases of both groups, and the average HR was recorded. Ratings of perceived exertion (RPE, scale of 6-20) [58] were assessed every 5 min.

\subsection{Procedure}

The participants were informed of the purpose of this study and asked to sign a consent form during the admission process.

The trial was approved by the institutional review board of Shanghai University of Sport.

Laboratory visits for participants were performed on two separate occasions at the same time of day.

During the baseline laboratory visit, the participants were asked to complete a demographics questionnaire, and their physical fitness (Table 1) was evaluated using a model the of Fitness Assessment System (BW-FC-9201L). All tests were repeated twice, and the best score was recorded. The participants were asked to performed the two cognitive tasks in a fixed sequence, starting with the Go/No-go test, under the guidance of experts.

During the second laboratory visit, the participants were equipped with an HR monitor throughout the session. After completing 30 min of either TC or HIIT, the participants were asked to perform cognitive tasks when their HR returned to $<10 \%$ of their pre-exercise HR. Figure 1 shows the flow diagram of the intervention processes of the two groups.

\subsection{Statistical analyses}

Continuous variables were analyzed by independent sample t-test for baseline comparison. All normally distributed variables were tested using the Kolmogorov-Smirnov test. The results of the Go/No-go test were analyzed by 2 (Time: baseline, post-test) $\times 2$ (Group: TC, HIIT) repeated-measures analysis of variance (ANOVA) to compare differences in Go RT under different conditions. Response accuracy was further analyzed using 2 (Time: baseline, post-test) $\times 2$ (Group: TC, HIIT) $\times 2$ (Condition: Go, No-go) repeated-measures ANOVA. RT and response accuracy in the Stroop test data were analyzed by using 2 (Time: baseline, post-test) $\times 2$ (Group: TC, HIIT) $\times 3$ (Condition: congruent, neutral, incongruent) repeatedmeasures ANOVA. A post-hoc test with Bonferroni adjustment was used for appropriate pairwise comparison if ANOVA showed a significant interaction. Trials with an RT of $<100$ ms were excluded from the calculation of participants' mean correct RT ( $n=1$ test excluded) because $<100 \mathrm{~ms}$ is considered insufficient for visual processing and stimulus response. All data are presented as the mean \pm standard deviation of the mean (SD). Statistical significance was set to a p-value $\leq 0.05$ according to Fisher statistics [59]. Additionally, the effect size (ES) of differences was analyzed using Neyman-Pearson statistics [60,61]. Statistical analyses were performed using SPSS 22.0 (Chicago, USA). 
Data that failed to pass the Kolmogorov-Smirnov test were subjected to the non-parametric test with Mann-Whitney U test.

\section{Results}

\subsection{Demographic}

The subjects in the two groups showed no significant differences in terms of age, height, weight, education level, years of drug use, and age of first drug use. While scores for one-leg stand with eyes closed differed between groups, differences in the general physical fitness of the two groups were not significant.

Table 1

Demographics of the participants at baseline

\begin{tabular}{|lllll|}
\hline & TC group & HIIT group & \multirow{2}{*}{ t-value } & p-value \\
\cline { 2 - 3 } & $\mathbf{( n = 2 4 )}$ & $\mathbf{( n = 2 3 )}$ & & \\
\hline Age (years) & $31.0(5.0)$ & $32.7(4.2)$ & -1.234 & 0.224 \\
\hline Height (cm) & $174.1(5.6)$ & $172.4(6.8)$ & 0.915 & 0.365 \\
\hline Weight (kg) & $77.6(7.5)$ & $78.0(11.9)$ & -0.153 & 0.879 \\
\hline BMI (kg/m $\left.{ }^{2}\right)$ & $25.6(2.4)$ & $26.2(3.6)$ & -0.681 & 0.131 \\
\hline Years of education (years) & $9.1(2.7)$ & $8.7(2.2)$ & 0.378 & 0.209 \\
\hline Years of drug use (years) & $6.8(4.3)$ & $9.0(4.8)$ & -1.622 & 0.112 \\
\hline Age of first drug use (years) & $25.1(5.0)$ & $25.7(4.6)$ & -0.435 & 0.666 \\
\hline Systolic Blood Pressure (mmHg) & $119.6(10.9)$ & $121.6(16.8)$ & -0.481 & 0.633 \\
\hline Diastolic Blood Pressure (mmHg) & $67.9(9.0)$ & $69.9(9.7)$ & -0.731 & 0.469 \\
\hline Vital capacity (ml) & $4548.0(928.5)$ & $4594.0(940.0)$ & -0.169 & 0.867 \\
\hline Hand grip (Kg) & $52.3(6.1)$ & $52.0(9.0)$ & 0.129 & 0.898 \\
\hline Push up (Repetitions) & $32.2(18.7)$ & $34.0(9.3)$ & -0.433 & 0.693 \\
\hline Sit-and-reach (cm) & $13.6(9.1)$ & $16.5(8.7)$ & -1.100 & 0.277 \\
\hline One-leg stand with eyes closed (s) & $47.2(32.9)$ & $30.0(20.8)$ & $2.174 *$ & $0.038 *$ \\
\hline Choice reaction time (ms) & $485.0(61.2)$ & $479.6(58.0)$ & 0.312 & 0.756 \\
\hline Data presented as mean (SD). TC, Tai chi; HIIT, High-intensity interval training. *p< 0.05. \\
\hline Co & & & \\
\hline
\end{tabular}




\subsubsection{Go/No-go test}

Independent $t$-test evaluating Go RT and Go/No-go accuracy revealed no significant differences between the two groups at the baseline. The descriptive statistical results are shown in Table 2.

The Go RT evaluated by two-way repeated-measures ANOVA revealed a statistically significant difference in the main effect of time $\left(F_{(1,44)}=8.424, p=0.006, \eta^{2}=0.161\right)$. The interaction between time and group was not significant. The post-hoc test showed that the Go RT of the post-test was significantly lower than that of the baseline. Additionally, the 2 (Time: baseline, post-test) $\times 2$ (Group: TC, HIIT) $\times 2$ (Condition: Go, No-go) of the accuracy with ANOVA revealed a statistically significant difference in the main effects of time $\left(F_{(1,44)}=7.813, p=0.008, \eta^{2}=0.151\right)$ and condition $\left(F_{(1,44)}=53.639, p<0.001, \eta^{2}=0.549\right)$. The posthoc test showed that the accuracy of the baseline was statistically significantly lower than that of the post-test and that the accuracy of the No-go condition is significantly lower than that of the Go condition. The interaction between time and condition revealed a statistically significant difference $\left(F_{(1,44)}=8.866, p\right.$ $=0.005, \eta^{2}=0.168$ ). Simple-effect analysis indicated that the accuracy of the baseline under the No-go condition is lower than that of the post-test $\left(F_{(1,44)}=9.793, p=0.003, \eta^{2}=0.182\right)$.

Table 2

Behavioral data for the Go/No-go task

\begin{tabular}{|lllll|}
\hline Variable & \multicolumn{2}{l}{ TC group $(\mathrm{n}=23)$} & \multicolumn{2}{l|}{ HIIT group $(\mathrm{n}=\mathbf{2 3})$} \\
\cline { 2 - 5 } & baseline & post-test & baseline & post-test \\
\hline Go accuracy $(\%)$ & $99.3 \pm 1.3$ & $99.3 \pm 1.0$ & $99.3 \pm 0.9$ & $99.4 \pm 1.3$ \\
\hline No-go accuracy (\%) & $96.2 \pm 2.8$ & $97.8 \pm 2.4$ & $96.0 \pm 3.8$ & $97.5 \pm 2.4$ \\
\hline Go RT (ms) & $400.9 \pm 57.5$ & $377.0 \pm 53.0$ & $386.0 \pm 40.5$ & $375.4 \pm 38.9$ \\
\hline Data presented as mean \pm SD. TC: Tai chi; HIIT: High-intensity interval training; RT: reaction time. \\
\hline
\end{tabular}

\subsubsection{Stroop test}

Independent $t$-test evaluating the RT and accuracy of the Stroop task under congruent, incongruent, and neutral conditions revealed no statistically significant differences between the two groups at the baseline. The descriptive statistical results are shown in Table 3.

The accuracy evaluated by three-way repeated-measures ANOVA revealed a significant difference in the main effects of time $\left(F_{(1,44)}=21.643, p<0.001, \eta^{2}=0.330\right)$ and condition $\left(F_{(2,88)}=8.598, p<0.001, \eta^{2}=\right.$ 0.163). The post-hoc test showed that the accuracy at the baseline is significantly lower than that of the post-test. Moreover, the accuracy of the incongruent condition was lower than those of the congruent and neutral conditions. The interaction between time and group revealed a significant difference $\left(F_{(1,44)}=\right.$ $4.162, p=0.047, \eta^{2}=0.086$ ). Further analysis indicated that the accuracy of the post-test is significantly higher than that of the baseline $\left(F_{(1,44)}=22.395, p<0.001, \eta^{2}=0.337\right)$ in the HIIT group. We further 
explored differences between the groups by conducting 2 (Time: baseline, post-test) $\times 2$ (Group: TC, HIIT) repeated-measures ANOVA; the results are shown in Fig. 2. The interaction between time and group was significant in neutral trials $\left(F_{(1,44)}=6.551, p=0.014, \eta^{2}=0.130\right)$, and a significant increase in accuracy was found in the HIIT group only in congruent trials $\left(F_{(1,44)}=5.395, p=0.025, \eta^{2}=0.109\right)$ by the post-hoc test.

The RT determined by three-way repeated-measures ANOVA revealed a significant difference between the main effects of time $\left(F_{(1,44)}=39.738, p<0.001, \eta^{2}=0.475\right)$ and condition $\left(F_{(2,88)}=73.684, p<0.001, \eta^{2}=\right.$ 0.626). In the post-hoc test, the RT of the post-test was significantly lower than that of the baseline, and the RT of the incongruent condition was significantly higher than those of the congruent and neutral conditions.

Table 3

Behavioral data for the Stroop task

\begin{tabular}{|lllll|}
\hline Variable & \multicolumn{2}{l}{ TC group(n=23) } & \multicolumn{2}{l|}{ HIIT group(n= 23) } \\
\cline { 2 - 5 } & baseline & post-test & baseline & post-test \\
\hline congruent accuracy (\%) & $91.7 \pm 4.4$ & $92.5 \pm 4.5$ & $92.1 \pm 4.8$ & $94.6 \pm 3.4$ \\
\hline incongruent accuracy (\%) & $88.6 \pm 6.2$ & $91.0 \pm 5.4$ & $89.5 \pm 6.1$ & $92.8 \pm 4.6$ \\
\hline neutral accuracy (\%) & $89.2 \pm 6.3$ & $90.7 \pm 5.2$ & $89.1 \pm 7.1$ & $95.2 \pm 3.7$ \\
\hline congruent RT (ms) & $604.0 \pm 52.8$ & $576.4 \pm 50.3$ & $591.3 \pm 53.0$ & $552.6 \pm 40.7$ \\
\hline incongruent RT (ms) & $634.2 \pm 50.7$ & $605.2 \pm 48.6$ & $623.0 \pm 50.9$ & $575.7 \pm 45.2$ \\
\hline neutral RT (ms) & $623.8 \pm 60.0$ & $602.2 \pm 46.3$ & $616.4 \pm 65.7$ & $567.7 \pm 44.9$ \\
\hline Data presented as mean \pm SD. TC: Tai chi; HIIT: High-intensity interval training; RT: reaction time. \\
\hline
\end{tabular}

\subsection{Exercise outcomes}

The overall average HRs of individuals with SUD during the TC and HIIT sessions were $120.69 \pm 7.64 \mathrm{bpm}$ and $147.75 \pm 20.71 \mathrm{bpm}$, respectively. The average HR range of individuals with SUD in the TC group was $55-75 \% \mathrm{HR}_{\max }$, which corresponds to the range of HRs following moderate-intensity exercise $(60-70 \%$ $\left.\mathrm{HR}_{\max }[55,62]\right)$. The average HR in the HIIT group during the running period was $165.31 \pm 12.44 \mathrm{bpm}$, which is $75-95 \% \mathrm{HR}_{\max }$. The average $\mathrm{HR}$ during the interval period was $130.20 \pm 6.27 \mathrm{bpm}$, which is 65$75 \% \mathrm{HR}_{\max }$. These values have been proved to elicit $90-95 \% \mathrm{HR}_{\max }$, which is consistent with the HIIT protocol [40]. The RPE scores observed during the TC and HIIT sessions were $12.69 \pm 1.32$ and $16.38 \pm$ 1.57 , respectively. Independent sample $t$-test revealed significant differences in exercise $H R(t=-3.878, p<$ $0.001)$ and RPE score $(t=-8.756, p<0.001)$ between the two groups, thus suggesting that the exercise sessions achieved the appropriate intensities. $\mathrm{HR}_{\max }$ values observed under each exercise type are shown in Fig. 3. 


\section{Discussion}

In this study, the acute effects of single sessions of TC and HIIT on inhibitory control were examined using behavioral measures during the Go/No-go and Stroop tasks. The findings indicated that TC and HIIT affect performance in the two cognitive tasks differently. In the Go/No-go test, acute TC and HIIT resulted in shorter RTs compared with the baseline and selectively increased response accuracy for incongruent trials. In the Stroop test, acute TC and HIIT resulted in shorter RTs under incongruent, neutral, and congruent conditions compared with the baseline. Moreover, the HIIT group showed superior improvements in the accuracy of cognitive inhibition under incongruent, neutral, and congruent conditions, while the TC group indicated improved accuracy only for incongruent trials. Thus, we summarize our findings under two main themes: (1) Improvements in inhibitory control and (2) additional cognitive benefits from HIIT.

\subsection{Improvements in inhibitory control}

The behavioral outcomes showed that the accuracy of both groups in the Go/No-go test improved and that RTs in the Go/No-go and Stroop tests were shorter in the number of trials that needed to be inhibited (under incongruent conditions). Inhibitory control is generally considered to be related to changes in RT and response accuracy under the incongruent conditions of the Go/No-go and Stroop tests [24, 63, 64]. The results demonstrated that both types of exercise lead to increased cognitive performance in SUD individuals, regardless of Go/No-go or Stroop congruency, thereby suggesting that TC and HIIT can improve the behavioral inhibition control of SUD individuals. This work is the first to provide evidence that TC and HIIT have similar benefits on the cognition of SUD individuals after acute exercise. TC is a Chinese traditional body-mind exercise. Although previous studies reported that TC is an exercise that can effectively improve the physical and mental health of SUD individuals [16-18], evidence of the ability of TC to improve the cognitive functions of individuals with SUD is unclear. The benefits of TC in elderly or cognitively impaired groups have been confirmed [65-67]. Previous research showed that long-term regular practice of TC could alleviate declines in the cognitive ability of the elderly [65]. However, one study found that TC does not improve the cognitive ability of the elderly [68]. Further research is needed to understand the differences in the boundary conditions of these contradictory effects. Because the intensity of TC was not monitored in the article [68] and may have been too low or too high, the results of cognitive improvement may not have reached statistical significance. In general, TC is considered a lowto-moderate-intensity exercise $[18,29,30]$ that results in $\operatorname{HR}_{\max }$ of approximately $50-70 \%[69,70]$. However, no comparison of the different exercise intensities of TC and the corresponding cognitive benefits is yet available. According to ACSM's Guidelines for Exercise Testing and Prescription [71], the $\mathrm{HR}$ range of low-intensity exercise is $57-64 \% \mathrm{HR}_{\max }$, that of moderate-intensity exercise is $64-76 \%$ $\mathrm{HR}_{\text {max }}$ and that of high-intensity exercise is $76-96 \% \mathrm{HR}_{\max }$. Thus, the intensity of the TC in our study can be considered moderate $\left(\mathrm{HR}\right.$ range $=55-75 \% \mathrm{HR}_{\max }$, average $\left.\mathrm{HR}=64 \% \mathrm{HR}_{\text {max }}\right)$. Earlier studies revealed that low-intensity exercise is not effective in improving cognitive function [14, 72]. Another meta-analysis indicated that moderate-intensity exercise of 45-60 min per session may be associated with cognitive 
benefits [28]. Overall, cognitive changes due to TC may be related to exercise intensity. Our study supports previous findings that moderate-intensity TC provides cognitive improvements in individuals with SUD and suggests that this exercise may be effective for improving cognitive conditions in SUD individuals.

In contrast to TC, HIIT is a vigorous exercise [45]. Some studies revealed that HIIT could improve the inhibition control of the cognitive impairment group [48-50]. Other studies have indicated that the inhibitory control benefits of HIIT and MICT are similar [73], consistent with our findings in this study. To date, few articles exploring cognitive inhibition control due to TC and other sports are available, and the current research cannot yet explain the advantages and disadvantages of moderate-intensity TC and other-intensity sports in detail. This article may help explain the difference between moderate- and highintensity exercises.

\subsection{Additional cognitive benefits from HIIT}

While no difference in RT was found between the two groups, the HIIT group showed superior improvements in cognitive accuracy under congruent and neutral conditions compared with the TC group in the Stroop task. By comparison, the accuracy of the TC group under these conditions simply indicated an upward trend. Given this finding, HIIT may be better able to improve cognitive accuracy than TC. However, the results do not show the difference between the two groups under incongruent conditions, and the accuracy rate of groups under incongruent conditions increased significantly in the post-hoc test. The increase in response accuracy under HIIT in congruent and neutral conditions may only indicate that HIIT results in better cognitive accuracy, rather than better inhibition control, given that inhibitory control is generally considered to be related to changes in RT and response accuracy under the incongruent condition of the Stroop test [64]. This work is the first to compare the cognitive effects of HIIT with TC, which are two completely different forms of exercise. Our study found that HIIT seems to be slightly better than TC in improving general cognitive accuracy. Although no direct evidence supports the results above, considering that TC is a moderate-intensity exercise, indirect evidence suggests similar behavioral outcomes [57, 73]. Exercise can improve the inhibition control of patients with SUD [14, 26]. Principally, the effects of different intensities of exercise seem to be different. According to the inverted U-shape relationship between exercise intensity and inhibition [26], moderate-intensity exercise appears to provide greater inhibition benefits than low- or high-intensity exercise. However, this general relationship does not consider the individual's initial conditions. Especially in individuals with SUD, the intensity of intervention could be highly dependent on the stage of recovery. In the laboratory setting, recent studies have found that HIIT tends to be more beneficial to inhibition control than MICT [57, 62]. Our results are consistent with a prior study [74] that showed that inverted-U theory is unsuitable for explaining the effects of HIIT. In brain-function studies, HIIT evoked cortical activation related to a Stroop interference on the left-dorsallateral prefrontal cortex, which corresponds with improved inhibitory control [75]. Furthermore, faster P3 latencies concurrent with smaller P3 amplitudes were found in HIIT compared with MICT, thereby indicating that HIIT may result in more efficient neuroelectric activation, as manifested by faster stimulus identification [62]. Long-term HIIT training can also improve exercise ability, metabolism, and cardiovascular health more effectively compared with MICT $[35,45]$. In summary, although HIIT showed 
inhibitory control benefits similar to those obtained from TC in this study, the former provides wider cognitive benefits, which suggests it is a better rehabilitation exercise for SUD.

Despite the benefits of exercise on cognition, other factors must also be considered for the practical applications of these exercises. Because the safety of HIIT is disputed [76], TC, the clinical safety of which is well established, may be a better rehabilitation exercise for special groups, such as SUD patients. Moreover, TC requires fewer training equipment than HIIT and, thus, is more suitable for large-scale management. Combining the results of this study, other factors affecting actual applications, and the different physical rehabilitation advantages brought about by the two exercises [17, 35, 45], we feel that TC is more suitable for the exercise rehabilitation of patients with SUD in the primary stage and that HIIT may be more suitable for the later stage of exercise rehabilitation. Both activities are feasible exercises that can be combined to contribute to the complete rehabilitation of patients with SUD.

The present study includes a number of limitations that may affect the generalizability of the results. Because we mainly relied on original Fisher statistics extended by ES according to Neyman-Pearson, generalizability cannot be established. The study groups only included male participants, which mean the observed benefits cannot be generalized to females or other cohorts. The cognitive tasks used in the study involved neutral task paradigms rather than substance-related task paradigms. Because substance abusers exhibit deficits with regard to inhibition, individuals who abuse drugs may exhibit more pronounced levels of activation in response to substance-related cues compared with neutral cues [77]. Another drawback of this study is the lack of a blank control group or low-intensity exercise group. Moreover, whether the effects in the present study are dependent on the type of movement or the intensity of the exercise requires further research. Future research may be combined with Electroencephalogram (EEG), functional near-infrared spectroscopy (fNIRS) or other neuroimaging techniques to explain the mechanism by which TC or HIIT intervention promotes cognitive function in individuals with SUD.

\section{Conclusion}

The present investigation demonstrated similar short-term facilitative effects on inhibitory control following TC and HIIT in patients with SUD. TC and HIIT can similarly promote the inhibitory control of individuals with SUD. Compared with the TC group, the HIIT group showed greater improvements in response accuracy. These findings demonstrate the potential applications of TC and HIIT in improving cognition in SUD. Both TC and HIIT are worth of being recommended as cognitive rehabilitation exercise for patients with SUD and the results encourage to continue the research according to the original interpretation of Fisher-statistics.

\section{Declarations}

\section{Ethics approval and consent to participate}


The study was conducted according to the guidelines of the Declaration of Helsinki, and approved by the Ethical Committees of the Shanghai University of Sport and the Shanghai Narcotics Control Commission (ChiCTR1900022158 Chinese Clinical Trial Registry: Registered 27th March, 2019.). All participants were informed about the purpose and nature of this study and provided written informed consent to be considered for participation in the study.

\section{Consent for publication}

$\mathrm{N} / \mathrm{A}$

\section{Availability of data and materials}

The data presented in this study will be available on request from the corresponding author and must be subject to the privacy restrictions.

\section{Competing interests}

The authors declare that there is no conflict of interest regarding the publication of this paper. The results of the study are presented clearly, honestly, and without fabrication, falsification, or inappropriate data manipulation.

\section{Funding}

This research was funded by Shanghai Drug Administration and National key research and development (2018YFC0807405).

\section{Authors' contributions}

Pang Xiaowu participated in the study design, data analysis and drafted the manuscript. Yang Suyong participated in the study design and manuscript revision. Xiao Ke participated in the coordination of intervention conducted in Shanghai Drug Compulsory Rehabilitation Center and established the appropriate experimental environment. Wang Tianyuan participated in the exercise intervention and data collection. Wang Jiabin participated in the exercise intervention and data collection. Wolfgang I. Schöllhorn participated in manuscript revision. Yin Yanqiang participated in the study design, data analysis and manuscript revision. Zhu Dong participated in the study design, manuscript revision and coordination of intervention conducted in Shanghai Drug Compulsory Rehabilitation Center. All authors reviewed the final manuscript.

\section{Acknowledgments}

This study was funded by Shanghai Drug Administration and National key research and development (2018YFC0807405). The study was supported by staffs of Shanghai Gao Jing mandatory detoxification and rehabilitation center. 


\section{Abbreviations}

SUD: Substance us disorder; TC: Tai chi; HIIT: High-intensity interval training; Meth: Methamphetamine; RT: Reaction time; MICT: Moderate-intensity continuous training; SCRC: Shanghai compulsory rehabilitation center; ES: Effect size; HR: Heart rate; EEG: Electroencephalogram; fNIRS: Functional nearinfrared spectroscopy.

\section{References}

1. Whiteford, H.A.; A.J. Ferrari; L. Degenhardt; V. FeiginT. Vos, The global burden of mental, neurological and substance use disorders: an analysis from the Global Burden of Disease Study 2010. PLoS One, 2015. 10(2): e0116820.

2. Borjkhani, M.; F. BahramiM. Janahmadi, Computational modeling of opioid-induced synaptic plasticity in hippocampus. PLoS One, 2018. 13(3): e0193410.

3. Tai, B.N.D. Volkow, Treatment for substance use disorder: opportunities and challenges under the affordable care act. Soc Work Public Health, 2013. 28(3-4): 165-74.

4. Bellamoli, E.; P. Manganotti; R.P. Schwartz; C. Rimondo; M. GommaG. Serpelloni, rTMS in the treatment of drug addiction: an update about human studies. Behav Neurol, 2014. 2014: 815215.

5. Scott, J.C.; S.P. Woods; G.E. Matt; R.A. Meyer; R.K. Heaton; J.H. Atkinson, et al., Neurocognitive Effects of Methamphetamine: A Critical Review and Meta-analysis. Neuropsychology Review, 2007. 17(3): 275-297.

6. D'Souza, M.S., Brain and Cognition for Addiction Medicine: From Prevention to Recovery Neural Substrates for Treatment of Psychostimulant-Induced Cognitive Deficits. Front Psychiatry, 2019. 10: 509.

7. Jones, H.W.; A.C. Dean; K.A. PriceE.D. London, Increased self-reported impulsivity in methamphetamine users maintaining drug abstinence. Am J Drug Alcohol Abuse, 2016. 42(5): 500506.

8. Smith, J.L.; R.P. Mattick; S.D. JamadarJ.M. Iredale, Deficits in behavioural inhibition in substance abuse and addiction: a meta-analysis. Drug Alcohol Depend, 2014. 145: 1-33.

9. Hulka, L.M.; M. Vonmoos; K.H. Preller; M.R. Baumgartner; E. Seifritz; A. Gamma, et al., Changes in cocaine consumption are associated with fluctuations in self-reported impulsivity and gambling decision-making. Psychol Med, 2015. 45(14): 3097-110.

10. Quednow, B.B.; K.U. Kuhn; C. Hoppe; J. Westheide; W. Maier; I. Daum, et al., Elevated impulsivity and impaired decision-making cognition in heavy users of MDMA ("Ecstasy"). Psychopharmacology (Berl), 2007. 189(4): 517-30.

11. Almeida, P.P.; G.M. de Araujo Filho; S.M. Malta; R.R. Laranjeira; A. Marques; R.A. Bressan, et al., Attention and memory deficits in crack-cocaine users persist over four weeks of abstinence. J Subst Abuse Treat, 2017. 81: 73-78. 
12. Wunderli, M.D.; M. Vonmoos; M. Furst; K. Schadelin; T. Kraemer; M.R. Baumgartner, et al., Discrete memory impairments in largely pure chronic users of MDMA. Eur Neuropsychopharmacol, 2017. 27(10): 987-999.

13. Lynch, W.J.; A.B. Peterson; V. Sanchez; J. AbelM.A. Smith, Exercise as a novel treatment for drug addiction: a neurobiological and stage-dependent hypothesis. Neurosci Biobehav Rev, 2013. 37(8): 1622-44.

14. Swenson, S.; K. Blum; T. McLaughlin; M.S. GoldP.K. Thanos, The therapeutic potential of exercise for neuropsychiatric diseases: A review. J Neurol Sci, 2020. 412: 116763.

15. Ashdown-Franks, G.; J. Firth; R. Carney; A.F. Carvalho; M. Hallgren; A. Koyanagi, et al., Exercise as Medicine for Mental and Substance Use Disorders: A Meta-review of the Benefits for Neuropsychiatric and Cognitive Outcomes. Sports Med, 2020. 50(1): 151-170.

16. Wang, D.; Y. Wang; Y. Wang; R. LiC. Zhou, Impact of Physical Exercise on Substance Use Disorders A Meta-Analysis. PLoS One, 2014. 9(10): e110728.

17. Zhu, D.; G. Dai; D. Xu; X. Xu; J. Geng; W. Zhu, et al., Long-Term Effects of Tai Chi Intervention on Sleep and Mental Health of Female Individuals With Dependence on Amphetamine-Type Stimulants. Front Psychol, 2018. 9: 1476.

18. Zhu, D.; D. Xu; G. Dai; F. Wang; X. XuD. Zhou, Beneficial effects of Tai Chi for amphetamine-type stimulant dependence: a pilot study. Am J Drug Alcohol Abuse, 2016. 42(4): 469-78.

19. Peterson, A.B.; J.M. AbelW.J. Lynch, Dose-dependent effects of wheel running on cocaine-seeking and prefrontal cortex Bdnf exon IV expression in rats. Psychopharmacology (Berl), 2014. 231(7): 1305-14.

20. Roberts, V.; R. Maddison; C. Simpson; C. BullenH. Prapavessis, The acute effects of exercise on cigarette cravings, withdrawal symptoms, affect, and smoking behaviour: systematic review update and meta-analysis. Psychopharmacology (Berl), 2012. 222(1): 1-15.

21. Lucas, S.J.; P.N. Ainslie; C.J. Murrell; K.N. Thomas; E.A. FranzJ.D. Cotter, Effect of age on exerciseinduced alterations in cognitive executive function: relationship to cerebral perfusion. Exp Gerontol, 2012. 47(8): 541-51.

22. Gimenez-Meseguer, J.; J. Tortosa-MartinezJ.M. Cortell-Tormo, The Benefits of Physical Exercise on Mental Disorders and Quality of Life in Substance Use Disorders Patients. Systematic Review and Meta-Analysis. Int J Environ Res Public Health, 2020. 17(10).

23. Chang, Y.K.; J.D. Labban; J.I. GapinJ.L. Etnier, The effects of acute exercise on cognitive performance: a meta-analysis. Brain Res, 2012. 1453: 87-101.

24. Wang, D.; C. ZhouY.K. Chang, Acute exercise ameliorates craving and inhibitory deficits in methamphetamine: An ERP study. Physiol Behav, 2015. 147: 38-46.

25. Colledge, F.; M. Gerber; U. PuhseS. Ludyga, Anaerobic Exercise Training in the Therapy of Substance Use Disorders: A Systematic Review. Front Psychiatry, 2018. 9: 644.

26. Wang, D.; C. Zhou; M. Zhao; X. WuY.K. Chang, Dose-response relationships between exercise intensity, cravings, and inhibitory control in methamphetamine dependence: An ERPs study. Drug Alcohol 
Depend, 2016. 161: 331-339.

27. Flemmen, G.; R. UnhjemE. Wang, High-Intensity Interval Training in Patients with Substance Use Disorder. BioMed Research International, 2014. 2014: 1-8.

28. Northey, J.M.; N. Cherbuin; K.L. Pumpa; D.J. SmeeB. Rattray, Exercise interventions for cognitive function in adults older than 50: a systematic review with meta-analysis. Br J Sports Med, 2018. 52(3): 154-160.

29. Hawkes, T.D.; W. ManselleM.H. Woollacott, Cross-sectional comparison of executive attention function in normally aging long-term T'ai chi, meditation, and aerobic fitness practitioners versus sedentary adults. J Altern Complement Med, 2014. 20(3): 178-84.

30. Pan, L.; J. Yan; Y. GuoJ. Yan, Effects of Tai Chi training on exercise capacity and quality of life in patients with chronic heart failure: a meta-analysis. Eur J Heart Fail, 2013. 15(3): 316-23.

31. Zhao, F.; C. ZhouT. Liu, Inhibition Effect and Its Potential Neurobiological Mechanism of Physical Exercise on Psychological Craving and Relapse Behaviors among Patients with Drug AddictionBased on the Regulation of Neurotransmitters, Hormones and Peptide through Physical Exercise. CHINA SPORT SCIENCE, 2018. 38(07): 33-41.

32. McGregor, G.; S. Nichols; T. Hamborg; L. Bryning; R. Tudor-Edwards; D. Markland, et al., High-intensity interval training versus moderate-intensity steady-state training in UK cardiac rehabilitation programmes (HIIT or MISS UK): study protocol for a multicentre randomised controlled trial and economic evaluation. BMJ Open, 2016. 6(11): e012843.

33. Onerup, A.; E. Angenete; D. Bock; M. Borjesson; M. Fagevik Olsen; E. Gryback Gillheimer, et al., The effect of pre- and post-operative physical activity on recovery after colorectal cancer surgery (PHYSSURG-C): study protocol for a randomised controlled trial. Trials, 2017. 18(1): 212.

34. Gibala, M.J.; J.P. Little; M.J. MacdonaldJ.A. Hawley, Physiological adaptations to low-volume, highintensity interval training in health and disease. J Physiol, 2012. 590(5): 1077-84.

35. Tabata, I.; K. Nishimura; M. Kouzaki; Y. Hirai; F. Ogita; M. Miyachi, et al., Effects of moderate-intensity endurance and high-intensity intermittent training on anaerobic capacity and V02max. Med Sci Sports Exerc, 1996. 28(10): 1327-30.

36. Schnyder, S., Handschin, C., Skeletal muscle as an endocrine organ: PGC-1 alpha, myokines and exercise. Bone, 2015. 80: 115-125.

37. Thompson, P.D.; B.A. Franklin; G.J. Balady; S.N. Blair; D. Corrado; N.A. Estes, 3rd, et al., Exercise and acute cardiovascular events placing the risks into perspective: a scientific statement from the American Heart Association Council on Nutrition, Physical Activity, and Metabolism and the Council on Clinical Cardiology. Circulation, 2007. 115(17): 2358-68.

38. Yang, W.P. Hu, Skeletal muscle regeneration is modulated by inflammation. J Orthop Translat, 2018. 13: 25-32.

39. Read, P.; J.L. Oliver; S.C.M.B.A. De; G.D. MyerR.S. Lloyd, Injury risk factors in male youth soccer players. Strength and Conditioning Journal 2015. 37: 1-7. 
40. Heisz, J.J.; M.G. Tejada; E.M. PaolucciC. Muir, Enjoyment for High-Intensity Interval Exercise Increases during the First Six Weeks of Training: Implications for Promoting Exercise Adherence in Sedentary Adults. PLoS One, 2016. 11(12): e0168534.

41. Currie, K.D.; J.B. Dubberley; R.S. McKelvieM.J. MacDonald, Low-volume, high-intensity interval training in patients with CAD. Med Sci Sports Exerc, 2013. 45(8): 1436-42.

42. Gillen, J.B.; B.J. Martin; M.J. Maclnnis; L.E. Skelly; M.A. TarnopolskyM.J. Gibala, Twelve Weeks of Sprint Interval Training Improves Indices of Cardiometabolic Health Similar to Traditional Endurance Training despite a Five-Fold Lower Exercise Volume and Time Commitment. PLoS One, 2016. 11(4): e0154075.

43. Helgerud, J.; K. Hoydal; E. Wang; T. Karlsen; P. Berg; M. Bjerkaas, et al., Aerobic high-intensity intervals improve VO2max more than moderate training. Med Sci Sports Exerc, 2007. 39(4): 665-71.

44. Macpherson, R.E., Hazell, T. J., Olver, T. D., Paterson, D. H., \& Lemon,P. W „, Run sprint interval training improves aerobic performance but not maximal cardiac output. Medicine \& Science in Sports\& Exercise, 2011. 43(1): 115-122.

45. Gayda, M.; P.A. Ribeiro; M. JuneauA. Nigam, Comparison of Different Forms of Exercise Training in Patients With Cardiac Disease: Where Does High-Intensity Interval Training Fit? Can J Cardiol, 2016. 32(4): 485-94.

46. Kodama, S.; K. Saito; S. Tanaka; M. Maki; Y. Yachi; M. Asumi, et al., Cardiorespiratory fitness as a quantitative predictor of all-cause mortality and cardiovascular events in healthy men and women a meta-analysis. JAMA - Journal of the American Medical Association, 2009. 301(19): 2024-2035.

47. Oberste, M.; P. Hartig; W. Bloch; B. Elsner; H.G. Predel; B. Ernst, et al., Control Group Paradigms in Studies Investigating Acute Effects of Exercise on Cognitive Performance-An Experiment on Expectation-Driven Placebo Effects. Front Hum Neurosci, 2017. 11: 600.

48. Fiorelli, C.M.; E.G. Ciolac; L. Simieli; F.A. Silva; B. Fernandes; G. Christofoletti, et al., Differential Acute Effect of High-Intensity Interval or Continuous Moderate Exercise on Cognition in Individuals With Parkinson's Disease. J Phys Act Health, 2019. 16(2): 157-164.

49. Baker, L.D.; L.L. Frank; K. Foster-Schubert; G.P. S.; C.W. Wilkinson; A. McTiernan, et al., Effects of aerobic exercise on mild cognitive impairment: A controlled trial. Arch Neurol, 2010. 67(1): 71-79.

50. Lautenschlager, N.T.; K.L. Cox; L. Flicker; J.K. Foster; F.M. van Bockxmeer; J. Xiao, et al., Effect of physical activity on cognitive function in older adults at risk for Alzheimer disease: A randomized trial. JAMA, 2008. 300(9): 1027-1037.

51. Chen, Y.; Y. Lu; C. ZhouX. Wang, The effects of aerobic exercise on working memory in methamphetamine-dependent patients: Evidence from combined fNIRS and ERP. Psychology of Sport and Exercise, 2020. 49.

52. Stroop, J.R., Studies of interference in serial verbal reactions. Journal of Experimental Psychology, 1935. 18(6): 643-662.

53. Dalley, J.W.; B.J. EverittT.W. Robbins, Impulsivity, compulsivity, and top-down cognitive control. Neuron, 2011. 69(4): 680-94. 
54. Wostmann, N.M.; D.S. Aichert; A. Costa; K. Rubia; H.J. MollerU. Ettinger, Reliability and plasticity of response inhibition and interference control. Brain Cogn, 2013. 81(1): 82-94.

55. Chu, C.H.; A.F. Kramer; T.F. Song; C.H. Wu; T.M. HungY.K. Chang, Acute Exercise and Neurocognitive Development in Preadolescents and Young Adults: An ERP Study. Neural Plast, 2017. 2017: 2631909.

56. Chu, C.-H.; B.L. Alderman; G.-X. WeiY.-K. Chang, Effects of acute aerobic exercise on motor response inhibition: An ERP study using the stop-signal task. Journal of Sport and Health Science, 2015. 4(1): 73-81.

57. Tsukamoto, H.; T. Suga; S. Takenaka; D. Tanaka; T. Takeuchi; T. Hamaoka, et al., Greater impact of acute high-intensity interval exercise on post-exercise executive function compared to moderateintensity continuous exercise. Physiol Behav, 2016. 155: 224-30.

58. Borg, G., Perceived exertion as an indicator of somatic stress. Scandinavian Journal of Rehabilitation Medicine, 1970. 2: 92-98.

59. Gigerenzer, G., Mindless Statistics. Journal of Socio - Economics, 2004. 33(5): 587-606.

60. Neyman, J.E.S. Pearson, On the Problem of the Most Efficient Tests of Statistical Hypotheses. 1992. 231(694-706): 289-337.

61. Nuzzo, R., Scientific method: Statistical errors. Nature, 2014: 150-152.

62. Kao, S.C.; D.R. Westfall; J. Soneson; B. GurdC.H. Hillman, Comparison of the acute effects of highintensity interval training and continuous aerobic walking on inhibitory control. Psychophysiology, 2017. 54(9): 1335-1345.

63. Yanagisawa, H.; I. Dan; D. Tsuzuki; M. Kato; M. Okamoto; Y. Kyutoku, et al., Acute moderate exercise elicits increased dorsolateral prefrontal activation and improves cognitive performance with Stroop test. NEUROIMAGE, 2010. 50(4): 1702-1710.

64. Cohen, J.D.; K. DunbarJ. Mcclelland, On the control of automatic processes: a parallel distributed processing account of the Stroop effect. Psychological Review, 1990. 97(3): 332-361.

65. Lam, L.C.; R.C. Chau; B.M. Wong; A.W. Fung; C.W. Tam; G.T. Leung, et al., A 1-year randomized controlled trial comparing mind body exercise (Tai Chi) with stretching and toning exercise on cognitive function in older Chinese adults at risk of cognitive decline. J Am Med Dir Assoc, 2012. 13(6): 568 e15-20.

66. Mortimer, J.A.; D. Ding; A.R. Borenstein; C. DeCarli; Q. Guo; Y. Wu, et al., Changes in brain volume and cognition in a randomized trial of exercise and social interaction in a community-based sample of non-demented Chinese elders. J Alzheimers Dis, 2012. 30(4): 757-66.

67. Yang, Y.; Y.L. Hao; W.J. Tian; L. Gong; K. Zhang; Q.G. Shi, et al., The effectiveness of Tai Chi for patients with Parkinson's disease: study protocol for a randomized controlled trial. Trials, 2015. 16: 111.

68. Gerritsen, R.J.S.; J. Lafeber; N. van den BeukelG.P.H. Band, No panacea? Tai Chi enhances motoric but not executive functioning in a normal aging population. Neuropsychol Dev Cogn B Aging Neuropsychol Cogn, 2020: 1-24. 
69. Cui, L.; H. Yin; S. Lyu; Q. Shen; Y. Wang; X. Li, et al., Tai Chi Chuan vs General Aerobic Exercise in Brain Plasticity: A Multimodal MRI Study. Sci Rep, 2019. 9(1): 17264.

70. Lan C, L.J., Chen SY, Wong MK, 12-month Tai Chi training in the elderly: its effect on health fitness. Medicine and Science in Sports and Exercise, 1998. 30: 345-351.

71. Thompson, P.D.; R. Arena; D. RiebeL.S. Pescatello, ACSM's new preparticipation health screening recommendations from ACSM's guidelines for exercise testing and prescription, ninth edition. Current Sports Medicine Reports, 2013. 12(4): 215.

72. McMorris, T.B.J. Hale, Differential effects of differing intensities of acute exercise on speed and accuracy of cognition: a meta-analytical investigation. Brain Cogn, 2012. 80(3): 338-51.

73. Kao, S.-C.; E.S. Drollette; J.P. Ritondale; N. KhanC.H. Hillman, The acute effects of high-intensity interval training and moderate-intensity continuous exercise on declarative memory and inhibitory control. Psychology of Sport and Exercise, 2018. 38: 90-99.

74. Alves, C.R.; V.H. Tessaro; L.A. Teixeira; K. Murakava; H. Roschel; B. Gualano, et al., Influence of acute high-intensity aerobic interval exercise bout on selective attention and short-term memory tasks. Percept Mot Skills, 2014. 118(1): 63-72.

75. Kujach, S.; K. Byun; K. Hyodo; K. Suwabe; T. Fukuie; R. Laskowski, et al., A transferable high-intensity intermittent exercise improves executive performance in association with dorsolateral prefrontal activation in young adults. Neuroimage, 2018. 169: 117-125.

76. Rognmo, O.; T. Moholdt; H. Bakken; T. Hole; P. Molstad; N.E. Myhr, et al., Cardiovascular risk of highversus moderate-intensity aerobic exercise in coronary heart disease patients. Circulation, 2012. 126(12): 1436-40.

77. Zamani, S.N., Mansouri, H., Fazilatpour, M., Shamsai, Z, A comparison of attentional bias towards drug cues in addicts and non-addicts. Int J High Risk Behav Addict, 2014. 3: e18669.

\section{Figures}



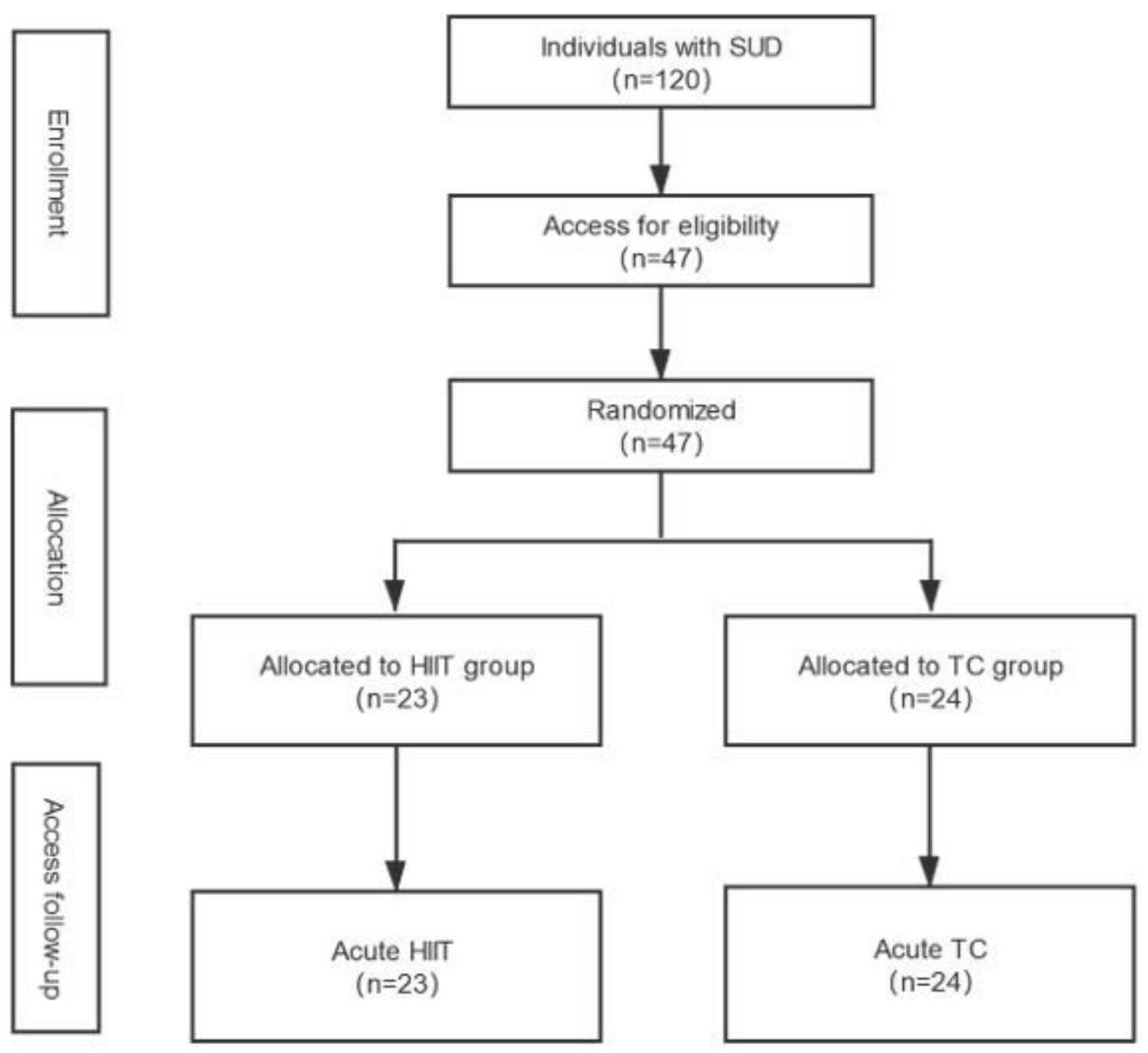

Figure 1

Flow diagram of the intervention process of the two groups. 

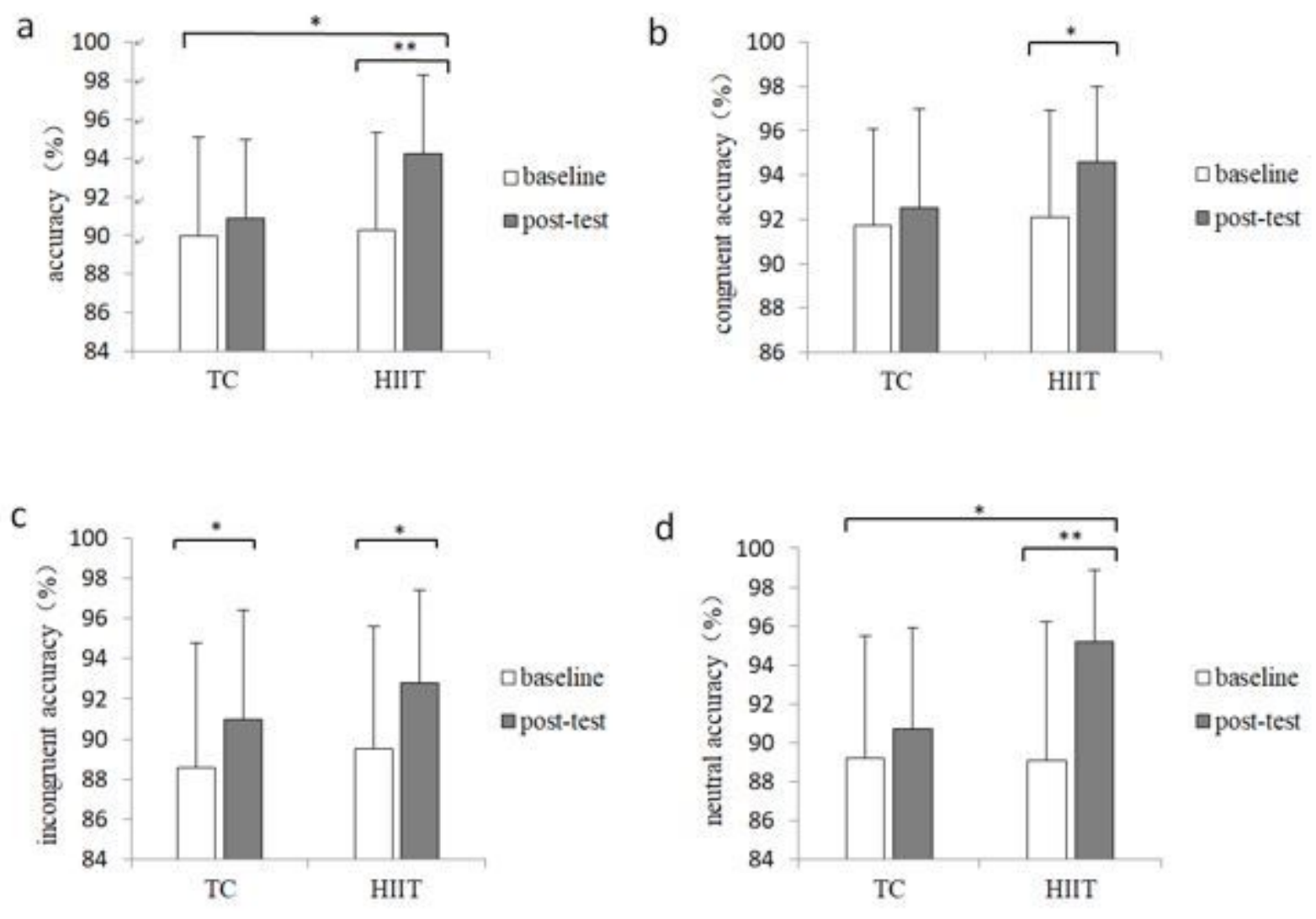

Figure 2

Changes in the four accuracy conditions of the Stroop test after TC and HIIT: (a) accuracy in all conditions, (b) congruent accuracy, (c) incongruent accuracy, and (d) neutral accuracy. ${ }^{\star} p<0.05,{ }^{*} p<$ 0.01. (a) shows the time $\times$ group result of accuracy under all conditions in $2 \times 2 \times 3$ repeated-measures ANOVA; (b), (c), and (d) show the time $\times$ group results of congruent accuracy, incongruent accuracy, and neutral accuracy in $2 \times 2$ repeated-measures ANOVA. 


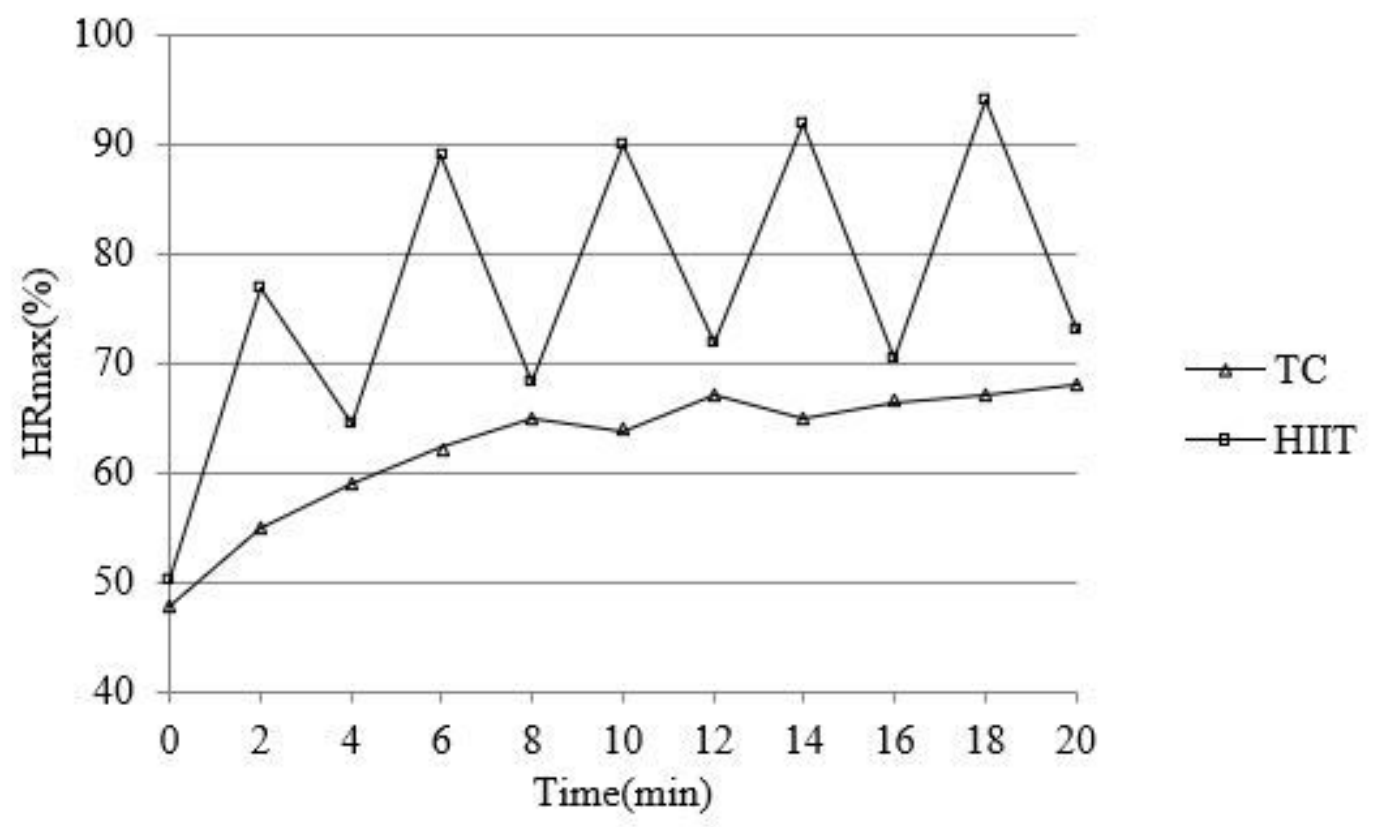

Figure 3

HRmax observed during each exercise condition. 